\title{
Role of Liver Transplantation for Hepatocellular Carcinoma
}

\author{
Vinay Kumaran \\ Liver Transplant and HPB Surgery, Kokilaben Dhirubhai Ambani Hospital, Mumbai, Maharashtra 400053, India
}

\begin{abstract}
This review evaluates the available evidence to establish the role of liver transplantation in the management of hepatocellular carcinoma in India. Most liver transplants in India are living donor transplants due to the paucity of brain dead organ donors. There is sufficient evidence to permit allocation of organs to patients with tumors within the Milan criteria. If the waiting list time is more than 6 months, a down-staging locoregional treatment modality such a trans-arterial chemoembolization, radiofrequency ablation, resection or percutaneous ethanol injection may be used to prevent disease progression. Allocating scarce livers to patients with more advanced tumors may not be justifiable. However, living donor liver transplantation may be offered to medically fit patients with hepatocellular carcinoma with cirrhosis, offering a guarded prognosis to patients beyond the Milan or UCSF criteria. Vascular invasion and extra-hepatic disease should be absolute contraindications to liver transplantation. (J Clin Exp Hepatol 2014;4:S97-S103)
\end{abstract}

$\mathrm{T}$ The role of liver transplantation in the management of hepatocellular carcinoma (HCC) is best understood in the context of the evolution of the modality. This is not a situation that lends itself to the conduct of multi-center double blind randomized controlled studies and much of the evidence comes from case series and database reviews. At the end of this review, it will be clear that patients with decompensated cirrhosis with small, not too numerous HCCs, with no vascular involvement and no extrahepatic spread are best served by an early liver transplant and that they do as well after liver transplant as patients transplanted for liver failure without HCC. It is also clear that patients with HCC infiltrating into major blood vessels or with extrahepatic spread have such poor outcomes that they are not candidates for liver transplant. Patients without cirrhosis with resectable HCC are obviously candidates for liver resection. Patients with early cirrhosis with small HCCs may be candidates for either resection or ablative therapies like radiofrequency ablation or for transplantation. Patients with larger HCCs, more numerous HCCs or adverse markers of tumor biology like markedly raised alfa-fetoprotein (AFP) levels or uptake

Keywords: hepatocellular carcinoma, liver transplantation, living donor liver transplantation, Milan criteria, UCSF criteria

Received: 20.5.2013; Accepted: 2.1.2014; Available online 24.1.2014

Address for correspondence: Vinay Kumaran, Head, Liver Transplant and HPB

Surgery, Kokilaben Dhirubhai Ambani Hospital, Mumbai, Maharashtra 400053, India. Tel.: +919022932994.

E-mail: kumaranvinay@yahoo.com

Abbreviations: AFP: alfa-fetoprotein; DDLT: deceased donor transplants; FDG: fluoro-deoxy-glucose; HCC: hepatocellular carcinoma; LDLT: living donor liver transplant; PET: positron emission tomography; TACE: transarterial chemoembolization

http://dx.doi.org/10.1016/j.jceh.2014.01.002 of fluoro-deoxy-glucose (FDG) on positron emission tomography (PET) have a higher recurrence rate and a poorer long-term survival after liver transplantation than patients transplanted for liver failure without HCC and it is not clear exactly where to draw the line. If the option of living donor liver transplant (LDLT) is available, the patient is not competing with another patient for a scarce resource (a deceased donor liver). However the potential benefit should be weighed in the context of the potential for harm to the donor. Whether to have different criteria for LDLT is an essentially ethical question and should perhaps be settled only after the donor's wishes are taken into account.

\section{EVOLUTION OF LIVER TRANSPLANTATION FOR HCC}

In the early years of liver transplantation, the procedure was a desperate attempt to save a dying patient. Neither the surgical technique nor the anesthetic management had been standardized. It was a victory for the patient to survive the operation and go home. Coagulopathy and bleeding was a major problem. ${ }^{1}$ In this context, the patient with an unresectable cancer in the liver was an attractive candidate. Since the cirrhosis was not very advanced, there would be less portal hypertension and coagulopathy and the patient was more likely to survive the operation. Liver transplantation was offered to patients with various unresectable malignancies in the liver. Of the first 7 liver transplants attempted, in Denver, Boston and Paris, 6 were for cancer, 3 HCC, 2 colorectal liver metastases and 1 cholangiocarcinoma. ${ }^{2}$ As liver transplantation evolved and the procedure became safer and more standardized, longterm survival became the norm. However, when the long- 
term survival of patients transplanted for liver cancer was evaluated, it was found to be dismal. In 1985, Starzl's group, which had by then moved to Pittsburgh, reported a $75 \%$ recurrence rate in patients transplanted for liver cancer. ${ }^{3}$ In contrast, patients with incidental HCCs diagnosed on pathological examination of the explanted liver did well with 12 of 13 alive without recurrence. Penn reported the results of transplantation for primary or metastatic cancer in 637 patients. ${ }^{4}$ The 5 -year survival of patients transplanted for HCC was a dismal $18 \%$. Patients transplanted for cirrhosis were doing well with improvements in immunosuppressive drugs and since there were not enough donor livers for the potential recipients, liver transplantation for HCC fell into disrepute for many years.

\section{The Milan Criteria}

In 1996, Mazzaferro et al from the University of Milan reported the outcomes in 48 patients with cirrhosis with small HCCs. ${ }^{5}$ Their criteria were a single tumor up to $5 \mathrm{~cm}$ in diameter or up to 3 tumors none of which was more than $3 \mathrm{~cm}$ in diameter. Twenty-eight patients with sufficient liver reserve underwent some treatment, predominantly transarterial chemoembolization (TACE) before transplant. After liver transplant patients were followed for a median of 26 months. The overall mortality was $17 \%$. The actuarial survival at 4 years was $75 \%$ with a recurrence free survival of $83 \%$. Thirty-five patients met the criteria at pathological examination as well $(73 \%)$ and had 4-year overall and recurrence free survivals of $85 \%$ and $92 \%$ respectively. The 13 patients who exceeded these limits on pathological examination had 4-year overall and disease free survivals of $50 \%$ and $59 \%$ respectively. The difference was highly significant $(P<0.01)$. It should be noted that only 60 of 295 patients referred for transplant for HCC met the criteria. Of these 1 died waiting for transplant, 11 were still waiting for organs and 48 had been transplanted. Transplant was performed a median of 143 days after staging.

The Milan criteria established that there is a category of patients with unresectable HCC against a background of cirrhosis who would do as well after transplant as patients transplanted for decompensated cirrhosis without HCC. The Milan criteria have been validated by many other centers. In a systematic review of such studies, Mazzaferro et al in 2011 looked at 90 studies spanning a period of 15 years and including 17,780 patients. ${ }^{6}$ Only $17 \%$ of the studies, including 1612 patients had level $1 \mathrm{~b}$ evidence. In 9 studies, patients who met Milan criteria and underwent liver transplant had post-transplant survival rates comparable to patients transplanted for non-tumor indications. Nineteen studies compared patients within Milan criteria and those beyond. Patients within Milan criteria had a better survival (hazard ratio 1.68, 95\% CI-1.39-2.03). When the studies were split according to the type of transplant, the hazard ratio was 1.76 (95\% CI-1.45-2.15) for deceased donor transplants (DDLT) while the advantage was considerably attenuated in LDLTs with a hazard ration of 1.28 with the $\mathrm{CI}$ beginning at 0.86 . This suggests that perhaps the wait for the organ in the DDLT situation may select patients with better tumor biology for transplant. ${ }^{7}$ It may also be that patients beyond Milan criteria progress while waiting for a deceased donor liver while they may have considerably superior outcomes in the LDLT scenario where this wait is eliminated.

\section{Beyond the Milan Criteria}

Obviously, everyone with tumors beyond the Milan criteria does not have recurrence after liver transplant and it seems unfair, for instance to condemn a patient with a $5.1 \mathrm{~cm} \mathrm{sin-}$ gle tumor to death. The possibility of seeing how far beyond the Milan criteria it is possible to go has been explored in many ways. The best known of the "beyond Milan" criteria are the University of California at San Francisco (UCSF) criteria. Yao et al reported 70 patients transplanted for HCC. ${ }^{8}$ They confirmed that the size limit for single tumors could be expanded to $6.5 \mathrm{~cm}$ and that for up to 3 tumors could be expanded to $4.5 \mathrm{~cm}$ provided the sum of the diameters of all the tumors was not more than $8 \mathrm{~cm}$. Patients within the UCSF criteria had 1 and 5 -year survivals of 90 and $75 \%$ while patients beyond the criteria had a 1 year survival of $50 \%(P=0.0005)$.

Patel et al analyzed data from the United Network for Organ Sharing (UNOS) database. ${ }^{9}$ From 2002 to 2007, 3434 patients were transplanted for HCC. Patients exceeding UCSF criteria, pediatric cases and patients whose size and number data was not available were excluded, leaving 1972 patients. Of these, 1913 patients were within the Milan criteria while 59 were beyond Milan but within the UCSF criteria. The survival of the two cohorts was similar, 1,2,3 and 4 year survival in the Milan cohort was $89 \%, 81 \%, 76 \%$ and $72 \%$ respectively while in the USCF cohort it was $91 \%, 80 \%, 68 \%$ and $51 \%$ respectively. This might be a better assessment of the impact of extending the criteria in the transplant population at large as opposed to a single center. While this report confirms the validity of the USCF criteria, it also illustrates the fact that only $3 \%$ of patients undergoing liver transplant for HCC will benefit from this extension of the criteria. In this study there were only 59 patients out of 1972 who were beyond the Milan criteria but within the UCSF criteria (2.9\%). However, it may be that the small number of patients beyond Milan and within UCSF might reflect the fact that many centers had not yet accepted the UCSF criteria as a replacement for the "gold standard" Milan criteria.

Toso et al suggested using the total tumor volume (TTV) instead of size and number to predict the risk of recurrence. ${ }^{10}$ However, this approach was limited by the 
fact that tumor volume was derived from the diameter rather than actually measured.

Mazzaferro et al from the Metroticket Study Investigator's Group reported data from a web based survey of patients beyond Milan criteria who underwent liver transplantation at multiple centers. ${ }^{11}$ Data for 1556 patients was collected from 36 centers. The concept of "Metroticket" comes from the idea that as one moves further from Milan (in terms of size or number of tumors), the cost (in terms of probability of recurrence) goes up. On explant pathology, 1112 patients had tumors beyond Milan criteria while 444 had tumors within Milan criteria. Microvascular invasion was found in $41 \%$ of patients beyond the Milan criteria. The 5-year overall survival for the patients beyond Milan criteria was $53.6 \%$ while that of patients within Milan criteria was $73.3 \%$. Patients without microvascular invasion and within what they term the "up to 7" criteria had a 5-year survival of $71.2 \%$ $(P<0.001)$. The sum of the size of the largest tumor (in $\mathrm{cm})$ and the number of tumors should not be more than 7. Unfortunately, the presence of microvascular invasion is not known before transplant.

Lee et al from the Asan Medical Center (AMC) reported their extended criteria. ${ }^{12}$ A largest tumor diameter of up to $5 \mathrm{~cm}$ and maximum number of tumors of up to 6 was permitted as long as there was no gross vascular invasion. Of 221 patients transplanted for HCC, 186 met these criteria, which was $10 \%$ more than the Milan criteria and 5.5\% more than the UCSF criteria. The 5-year survival rates were $76 \%$ and $44.5 \%$ within and beyond the Milan criteria, $75.9 \%$ and $36.4 \%$ within and beyond the UCSF criteria and $76.3 \%$ and $18.9 \%$ within and beyond the AMC criteria $(P<0.001)$. However, theirs was a very homogenous population (more than $90 \%$ due to hepatitis B) and it is not clear if these criteria can be extrapolated to Indian and Western populations where there is a large proportion of hepatitis $\mathrm{C}$, nonalcoholic steatohepatitis (NASH), alcoholic and cryptogenic liver disease.

The major "criteria" used for risk stratification of patients with cirrhosis with hepatocellular carcinoma undergoing liver transplantation are compared in Table 1.

\section{Beyond Tumor Size and Number}

While size and number do seem to be surrogate markers of biological behavior of HCC it is increasingly evident that these alone are not good enough to differentiate between tumors with favorable outcomes and those that disseminate early. For instance, since HCC is asymptomatic, a biologically indolent tumor may attain a large size before being detected but may still have a more favorable prognosis than a small aggressive tumor that has invaded small portal vein radicles and produced 2 metastatic lesions.

Ito et al from Kyoto University reported on expanded criteria in their patients undergoing LDLT for HCC. ${ }^{13}$ Of their 125 patients, 70 were within the Milan criteria while 55 were beyond. They reported that patients beyond the Milan criteria but with $\leq 10$ tumors all under $5 \mathrm{~cm}$ in diameter $(n=30)$ had 5 -year recurrence rates $(7.3 \%)$ similar to those of patients within the Milan criteria. They incorporated the levels of Protein Induced by Vitamin K Absence or Antagonist II (PIVKA II) and defined their own criteria: up to 10 tumors, none more than $5 \mathrm{~cm}$ in diameter and PIVKA II levels up to $400 \mathrm{mAU} / \mathrm{ml}$. The 78 patients who met these Kyoto criteria had a 5-year survival of $86.7 \%$. These criteria were further validated by the same group in $2013^{14}$ in a series of 198 patients. Patients within the Kyoto criteria (147) had a 5-year survival of $82 \%$ while the 49 patients exceeding them had a 5-year survival of $42 \%(P<0.001)$.

The International Union Against Cancer (UICC) TNM staging has not been useful for assessing prognosis. ${ }^{15}$ Marsh et al from the Thomas E. Starzl Transplantation Institute reported 307 patients undergoing liver transplant for HCC. ${ }^{16}$ They found depth of vascular invasion, lobar distribution, lymph node status and the largest tumor size to be independent predictors of prognosis. Tumor number was a prognostic factor on multivariate analysis. They proposed a modification of the staging system.

Yao et al compared the then proposed UCSF criteria with the Milan criteria and the Pittsburgh criteria in 70 patients transplanted for HCC. ${ }^{17}$ The difference in survival between 24 patients beyond Milan criteria and 46 patients within Milan criteria was not significant. Fourteen patients (20\%) exceeded the Milan criteria but met the UCSF criteria

Table 1 Criteria for Risk of Recurrence After Liver Transplant for HCC.

\begin{tabular}{|c|c|c|c|c|c|c|c|}
\hline \multirow[t]{2}{*}{ Criteria } & \multirow[t]{2}{*}{ Year } & \multirow[t]{2}{*}{ Number of patients } & \multirow[t]{2}{*}{ Size of tumor $(\mathrm{cm})$} & \multirow[t]{2}{*}{ Number of tumors } & \multirow[t]{2}{*}{ Others } & \multicolumn{2}{|c|}{ Survival } \\
\hline & & & & & & Within & Beyond \\
\hline \multirow[t]{2}{*}{ Milan $^{5}$} & 1996 & 48 & $\leq 5$ & 1 & & $75 \%$ & $50 \%$ \\
\hline & & & $\leq 3$ & 3 & & & \\
\hline \multirow[t]{2}{*}{ UCSF $^{8}$} & 2001 & 70 & $\leq 6.5$ & 1 & & $75 \%$ & $50 \%$ \\
\hline & & & $\leq 4.5$ & $\leq 3$ & & & \\
\hline Asan Medical Center ${ }^{12}$ & 2008 & 221 & $\leq 5$ & $\leq 5$ & & $76.3 \%$ & $18.9 \%$ \\
\hline Up-to $7^{11}$ & 2009 & 1556 & Size $+n$ & mber $\leq 7$ & No microvascular invasion & $71.2 \%$ & $48.1 \%$ \\
\hline
\end{tabular}


and had a 2-year survival of $86 \%$. Patients with Pittsburgh stage 1,2 and $3 \mathrm{~A}$ had survival similar to patients within UCSF criteria and significantly better than patients with Pittsburgh stage $3 \mathrm{~B}$ and 4 . The Pittsburgh criteria, unfortunately, require information about vascular invasion and lymph node involvement, which is not available before transplant.

Poon et al from the University of Hong Kong reported that hepatitis $\mathrm{C}$ and microscopic venous invasion, in addition to tumor size and multifocality were independent predictors of recurrence in 247 cirrhotic patients undergoing resection or transplantation. ${ }^{18}$

The alfa-fetoprotein level (AFP) has been used as a surrogate marker for vascular involvement and high risk for recurrence. The Asan Medical Center group reported that all patients with very high AFP levels (above $3000 \mathrm{ng} / \mathrm{ml}$ ) had recurrence within 3 years. ${ }^{12}$ Toso et al looked at available data from the Scientific Registry of Transplant Recipients (SRTR) on 6478 patients being transplanted for HCC. ${ }^{19}$ They reported that total tumor volume (TTV) and AFP were independent predictors of recurrence. They developed a composite score where patients with a TTV of more than $115 \mathrm{cc}$ or an AFP level more than $400 \mathrm{ng} /$ $\mathrm{ml}$ were outside criteria. Patients beyond these criteria had a survival of less than $50 \%$ at 3 years. One must be guarded while interpreting these data because being a database study, patients alive with recurrence were missed. The imaging was done an average of 3.6 months before the transplant and the TTV was calculated from the diameter of the tumor assuming each tumor to be a sphere and not actually measured.

Wei et al used microarrays to compare gene expression profiles of HCC tissues with non-tumorous tissues of the same livers. ${ }^{20}$ They found that 509 genes (about 6\% of the total) were differentially expressed between HCC and non-tumor tissue. Two hundred were down regulated while 307 were up regulated. These genetic pathways would be targets for differential expression at different stages of HCC and to try and differentiate between biologically aggressive tumors and indolent ones. While this approach seems promising, it would require access to tumor tissue before therapy can be started.

\section{THE ROLE OF LIVING DONOR LIVER TRANSPLANTATION}

LDLT is an attractive option for patients with HCC, particularly for those beyond the conventional criteria who would not be listed for a DDLT. However, the risk to the donor becomes a factor in this situation. Liver donation, particularly right lobe donation (required for most adult to adult LDLTs) is associated with definite donor mortality and significant morbidity. The mortality of liver donation has been reported to be about $0.2 \%$ (probably an underestimate since there is no registry in many countries which perform LDLT) and the morbidity has been estimated to be $0-100 \%$ depending on how it is measured. ${ }^{21}$ It is also clear that as center experience grows, the incidence of serious complications decreases. ${ }^{22}$ The risk to the donor would not be justifiable if there is little possibility of the recipient surviving long-term as when there is extrahepatic disease or gross vascular invasion. However, between the conventional criteria and these patients is a significant population of patients who would benefit from liver transplantation although with a relatively high-risk of tumor recurrence. The concept of "double equipoise" describes the balance between the benefit to the recipient and the risk to the donor. ${ }^{23}$ Assuming that the donor risk is fixed, an LDLT is easy to justify when the outcomes are known to be good as with a recipient whose tumor is within the Milan criteria in a milieu where there is a shortage of livers. As the risk of recurrence goes up (with increasing tumor size, number, AFP level etc), the risk to the donor becomes more problematic. Certainly, everyone would agree that LDLT should not be offered to a recipient with extrahepatic disease or macrovascular invasion but the situation where the tumor is beyond the convention criteria and a recurrence rate of approximately $50 \%$ can be expected in the longterm it is unclear where the line should be drawn. It is, in fact, not even clear if the surgeon has a right to be paternalistic and draw a line if a well-informed donor wishes to give the recipient a chance to receive the only potentially curative treatment available.

Even in the US where costs are high and there is a very small LDLT component in a predominantly DDLT scenario, it has been established that introducing the option of LDLT improves survival though at a higher cost. ${ }^{24}$ Even in small HCC, LDLT seems to be more effective from a life-expectancy and cost effectiveness viewpoint ${ }^{25}$ and certainly both donor and recipient outcomes have improved in the 12 years that have passed since the publication of this analysis. The Mount Sinai group reported outcomes of offering LDLT to patients beyond the Milan criteria. ${ }^{26}$ Thirty-six patients with HCC underwent liver transplant with a follow-up of more than one year. The mean wait for LDLT was 62 days (surprisingly long, the corresponding time at an Indian LDLT center would be about 14 days) while the wait for DDLT during the same period was 459 days. Fifty-three percent of the patients exceeded UNOS priority criteria. One and 2-year survivals were $75 \%$ and $60 \%$ respectively and there was no significant difference in survival between patients within ${ }^{24}$ and beyond the criteria. ${ }^{12}$ It must be noted that the duration of follow up in this study was short and the number of patients beyond criteria was small. The University Hospital, Essen reported a similar initial experience. ${ }^{27}$ Of their $34 \mathrm{pa}-$ tients, 19 met the Milan criteria while 15 exceeded them. Overall survival at 1 and 2 years was $68 \%$ and $62 \%$ respectively. It should be noted that both these series had a higher mortality from non-tumor causes than would be 
expected at a high volume LDLT center now and this may have further obscured the role of tumor stage but they certainly demonstrate that a policy of offering LDLT to patients even beyond the Milan criteria does not result in unacceptably poor outcomes. Todo and Furukawa in 2004, reported the Japanese experience with LDLT for HCC. ${ }^{28}$ There were 316 living donor liver transplants across 29 centers with a median follow-up of 16 months. Overall survival was $74.7 \%$. One and 3-year survivals were $78.1 \%$ and $69 \%$ respectively. The recurrence rate was $12.7 \%$ indicating again that much of the mortality was due to other causes. For patients within the Milan criteria, overall and recurrence free survival at 3 years were $78.7 \%$ and $79.1 \%$ respectively. Patients beyond Milan criteria had 60.4 and 52.6\% respectively. Interestingly, the Milan criteria were used to deny LDLT at only one third of the centers. The others considered patients beyond the Milan criteria on a caseby-case basis but all centers excluded patients with major vascular invasion or extrahepatic disease.

Our own experience with 77 patients undergoing LDLT for HCC was reported in $2010 .^{29}$ Forty patients were within the UCSF criteria, 28 were beyond and 9 were not classifiable because of inadequate documentation of size or number of tumors. The 1 and 3 year recurrence-free survival in patients within and beyond the criteria were $92.4 \%$ and $83 \%$ versus $96.4 \%$ and $76.4 \%$ at a median follow-up of 589 days. Later, after a median follow-up of 989 days, the overall survival was $84.3 \%$ (unpublished data). The 1-, 3- and 5-year survival for the entire cohort was $92.9 \%, 84.6 \%$ and $78.9 \%$ respectively. The overall, 1-, 2- and 3-year survival in patients within the UCSF criteria was $91.3 \%, 97.8 \%, 93.1 \%$ and $89.6 \%$ respectively while the corresponding figures for patients beyond UCSF criteria were $65 \%, 80 \%, 69.6 \%$ and $69.6 \%$ respectively.

\section{DOWN-STAGING FOR LIVER TRANSPLANT}

The rationale for down-staging HCC while waiting for liver transplant is two-fold: to reduce the chances of disease progression while waiting for a liver and to reduce the disease burden to within the criteria used for listing. The response to down-staging may also be a surrogate marker of the biological behavior of the tumor.

Yao et $\mathrm{al}^{30}$ reported outcomes of down-staging for 61 patients slightly beyond Milan criteria. They took patients with single lesions between 5 and $8 \mathrm{~cm}$ or $2-3$ lesions with one lesion exceeding $3 \mathrm{~cm}$ or $4-5$ lesions with none more than $3 \mathrm{~cm}$ and total diameter up to $8 \mathrm{~cm}$ (note that most of these are within the UCSF criteria to begin with) and subjected the tumors to locoregional therapy. The tumor was down-staged to within Milan criteria in 43 patients (70.5\%). Treatment failure occurred in 18 patients (29.5\%). Thirty-five patients were transplanted and had 1and 4-year survivals of $96.2 \%$ and $92 \%$ respectively.

Llovet et al from Barcelona reported a Markov model analysis of the benefits of percutaneous alcohol injection or resection in patients with cirrhosis and HCC listed for liver transplant. ${ }^{31}$ Liver resection was considered only in Childs-Pugh A patients while percutaneous treatment was considered for B patients as well. Surgical resection increased the transplantation rate by more than $10 \%$ and increased the life-expectancy by 4.8-6.1 months at a cost of $\$ 40,000 /-$ per life year gained if the waiting time was more than 1 year. For shorter waiting times the cost was $\$ 74,000 /-$ per life year gained. Percutaneous treatment increased the life-expectancy by 5.2-6.7 months at a cost of $\$ 20,000 /-$ per life year gained with all waiting times. Millonig et al from Innsbruck, Austria reported the outcomes of a policy of using TACE to suppress or down-stage HCC while waiting for DDLT. ${ }^{32}$ Chemoembolization was performed in 116 of 175 patients eligible for liver transplant (on the basis of hypervascularity on CT). Ten patients progressed while waiting for the liver and were removed from the list. Patients whose tumors progressed beyond the UCSF criteria were taken off the list. The remaining 106 patients were transplanted. The median waiting time for transplant was 9 months. Sixty-eight patients (58.6\%) were within the Milan criteria, 33 were beyond the Milan criteria but within the UCSF criteria (28.5\%) and 15 were beyond the UCSF criteria but were down-staged to within the UCSF criteria. According to radiological assessment of response to TACE, there were 33 (28.4\%) complete responses, $66(56.9 \%)$ partial responses, 3 (2.6\%) stable diseases and $13(11.2 \%)$ with progression of disease. The dropout rate was $2.9 \%$ for those within Milan criteria, $12.1 \%$ for those beyond Milan but within UCSF criteria and $26.7 \%$ for those down-staged to within UCSF criteria. Patients with complete or partial response had 1,2 and 5 year actuarial survival after liver transplant of $100 \%$, 93.2\% and $85.7 \%$ respectively while those who did not respond or progressed had corresponding survivals of $82.4 \%, 50.7 \%$ and $19.3 \%$ respectively. Vitale et al from Padova, Italy used an institutional algorithm for treatment of HCC before liver transplantation. ${ }^{33}$ They prioritized patients who did not respond to treatment for transplant. Response to therapy was the sole predictor of dropout but those which were actually transplanted from the non-responders group had a similar survival although with a somewhat higher recurrence rate. In a review of the available evidence, Cescon et $\mathrm{al}^{34}$ recommended no locoregional therapy for patients with T1 tumors with waiting times less than 6 months and treatment based on the BCLC system for T2 tumors and waiting times more than 6 months. No recommendation could be made for down-staging strategies because of paucity of evidence.

\section{SUMMARY AND RECOMMENDATIONS}

As we noted in the beginning, there will never be a randomized, double blind controlled study comparing liver transplantation with anything else as a treatment for HCC and 
the results of retrospective data speak for themselves. We may summarize the evidence available thus:

1. Patients with hepatocellular carcinoma and cirrhosis meeting the Milan criteria should be offered liver transplantation. The outcomes are comparable to patients being transplanted for benign indications. Level of evidence 1b. Recommendation grade A.

2. Some patients beyond the Milan criteria but within other criteria such as the UCSF, TTV or AMC criteria may be offered liver transplantation with similar outcomes. However, this population will be a small proportion of the patients beyond the Milan criteria. Level of evidence $2 \mathrm{~b}$. Recommendation grade $\mathrm{B}$.

3. Patients beyond these conventional criteria but without macrovascular invasion or extrahepatic disease have a long-term survival of about $50 \%$. They should not be offered DDLT as long as there is a disparity between the requirement of livers and the supply. Level of evidence $1 \mathrm{~b}$. Recommendation grade A. They may be offered LDLT with a guarded prognosis after extensive counseling of the donor and discussion of alternative treatment options on a case-by-case basis. Downstaging may be an option for patients who do not have live donors but policy regarding listing of such patients should be developed by the transplant listing authority based on the availability of deceased donor organs in that state.

\section{CONFLICTS OF INTEREST}

The author has none to declare.

\section{REFERENCES}

1. Starzl TE, Marchioro TL, Von Kaulla KN, Hermann G, Brittain RS, Waddell WR. Homotransplantation of the liver in humans. Surg Gynecol Obstet. 1963;117:659-676.

2. Starzl TE. The saga of liver replacement, with particular reference to the reciprocal influence of liver and kidney transplantation (19551967). J Am Coll Surg. 2002;195(5):587-610.

3. Iwatsuki S, Gordon RD, Shaw Jr BW, Starzl TE. Role of liver transplantation in cancer therapy. Ann Surg. 1985;202(4):401-407.

4. Penn I. Hepatic transplantation for primary and metastatic cancers of the liver. Surgery. 1991;110(4):726-734.

5. Mazzaferro V, Regalia E, Doci R, et al. Liver transplantation for the treatment of small hepatocellular carcinomas in patients with cirrhosis. N Engl J Med. 1996;334(11):693-699.

6. Mazzaferro V, Bhoori S, Sposito C, et al. Milan criteria in liver transplantation for hepatocellular carcinoma: an evidence-based analysis of 15 years of experience. Liver Transpl. 2011;17(suppl 2):S44-S57.

7. Grant D, Fisher RA, Abecassis M, McCaughan G, Wright L, Fan ST. Should the liver transplant criteria for hepatocellular carcinoma be different for deceased donation and living donation? Liver Transplant. 2011;17(suppl 2):S133-S138.

8. Yao FY, Ferrell L, Bass NM, et al. Liver transplantation for hepatocellular carcinoma: expansion of the tumor size limits does not adversely impact survival. Hepatology. 2001;33(6):1394-1403.
9. Patel SS, Arrington AK, McKenzie S, et al. Milan criteria and UCSF criteria: a preliminary comparative study of liver transplantation outcomes in the United States. Int J Hepatol. 2012;2012:253517. http://dx.doi.org/10.1155/2012/253517. Epub 2012 Aug 22.

10. Toso C, Trotter J, Wei A, et al. Total tumor volume predicts risk of recurrence following liver transplantation in patients with hepatocellular carcinoma. Liver Transpl. 2008;14(8):1107-1115.

11. Mazzaferro V, Llovet JM, Miceli R, et al. Predicting survival after liver transplantation in patients with hepatocellular carcinoma beyond the Milan criteria: a retrospective, exploratory analysis. Lancet Oncol. 2009;10(1):35-43.

12. Lee SG, Hwang S, Moon DB, et al. Expanded indication criteria of living donor liver transplantation for hepatocellular carcinoma at one large-volume center. Liver Transpl. 2008;14(7):935-945.

13. Ito $\mathrm{T}$, Takada $\mathrm{Y}$, Ueda $\mathrm{M}$, et al. Expansion of selection criteria for patients with hepatocellular carcinoma in living donor liver trans plantation. Liver Transplant. 2007;13:1637-1644.

14. Kaido T, Ogawa K, Mori A, et al. Usefulness of the Kyoto criteria as expanded selection criteria for liver transplantation for hepatocellular carcinoma. Surgery. 2013;154:1053-1060.

15. Izumi R, Shimizu K, li T, et al. Prognostic factors of hepatocellular carcinoma in patients undergoing hepatic resection. Gastroenterology. 1994;106(3):720-727.

16. Marsh JW, Dvorchik I, Bonham CA, Iwatsuki S. Is the pathologic TNM staging system for patients with hepatoma predictive of outcome? Cancer. 2000;88(3):538-543.

17. Yao FY, Ferrell L, Bass NM, Bacchetti P, Ascher NL, Roberts JP. Liver transplantation for hepatocellular carcinoma: comparison of the proposed UCSF criteria with the Milan criteria and the Pittsburgh modified TNM criteria. Liver Transpl. 2002;8(9):765-774.

18. Poon RT, Fan ST, Lo CM, Liu CL, Wong J. Difference in tumor invasiveness in cirrhotic patients with hepatocellular carcinoma fulfilling the Milan criteria treated by resection and transplantation: impact on long-term survival. Ann Surg. 2007;245(1):51-58.

19. Toso C, Asthana S, Bigam DL, Shapiro AM, Kneteman NM. Reassessing selection criteria prior to liver transplantation for hepatocellular carcinoma utilizing the Scientific Registry of Transplant Recipients database. Hepatology. 2009;49(3):832-838.

20. Wang W, Peng JX, Yang JQ, Yang LY. Identification of gene expression profiling in hepatocellular carcinoma using cDNA microarrays. Dig Dis Sci. 2009;54(12):2729-2735.

21. Middleton PF, Duffield M, Lynch SV, et al. Living donor liver transplantation-adult donor outcomes: a systematic review. Liver Transpl. 2006;12(1):24-30.

22. Azoulay D, Bhangui P, Andreani P, et al. Short- and long-term donor morbidity in right lobe living donor liver transplantation: 91 consecutive cases in a European Center. Am J Transpl. 2011;11(1): 101-110.

23. Pomfret EA, Lodge JP, Villamil FG, Siegler M. Should we use living donor grafts for patients with hepatocellular carcinoma? Ethical considerations. Liver Transplant. 2011;17:S128-S132.

24. Northup PG, Abecassis MM, Englesbe MJ, et al, Group, Adult-to-Adult Living Donor Liver Transplantation Cohort Study. Addition of adult-toadult living donation to liver transplant programs improves survival but at an increased cost. Liver Transpl. 2009;15(2):148-162.

25. Sarasin FP, Majno PE, Llovet JM, Bruix J, Mentha G, Hadengue A. Living donor liver transplantation for early hepatocellular carcinoma: a life-expectancy and cost-effectiveness perspective. Hepatology. 2001;33(5):1073-1079.

26. Gondolesi GE, Roayaie S, Muñoz L, et al. Adult living donor liver transplantation for patients with hepatocellular carcinoma: extending UNOS priority criteria. Ann Surg. 2004;239(2):142-149.

27. Malagó M, Sotiropoulos GC, Nadalin S, et al. Living donor liver transplantation for hepatocellular carcinoma: a single-center preliminary report. Liver Transpl. 2006;12(6):934-940. 
28. Todo S, Furukawa H, Transplantation, Japanese Study Group on Organ. Living donor liver transplantation for adult patients with hepatocellular carcinoma: experience in Japan. Ann Surg. 2004;240(3): 451-459.

29. Kumaran V, Singh T, Mohanka R, et al. Living donor liver transplantation for hepatocellular carcinoma: tumour size and number may not matter. Liver Transplant. 2010;16(6 suppl 1):S186.

30. Yao FY, Kerlan Jr RK, Hirose R, et al. Excellent outcome following downstaging of hepatocellular carcinoma prior to liver transplantation: an intention-to-treat analysis. Hepatology. 2008;48:819-827.

31. Llovet JM, Mas X, Aponte JJ, et al. Cost effectiveness of adjuvant therapy for hepatocellular carcinoma during the waiting list for liver transplantation. Gut. 2002;50(1):123-128.
32. Millonig G, Graziadei IW, Freund MC, et al. Response to preoperative chemoembolization correlates with outcome after liver transplantation in patients with hepatocellular carcinoma. Liver Transpl. 2007;13(2):272-279.

33. Vitale A, D'Amico F, Frigo AC, et al. Response to therapy as a criterion for awarding priority to patients with hepatocellular carcinoma awaiting liver transplantation. Ann Surg Oncol. 2010;17(9): 2290-2302.

34. Cescon M, Cucchetti A, Ravaioli M, Pinna AD. Hepatocellular carcinoma locoregional therapies for patients in the waiting list. Impact on transplantability and recurrence rate. J Hepatol. 2013;58(3): 609-618. 